\title{
CASES DEMONSTRATED AT THE F.R.C.S. (FINAL) CLASS.
}

\section{A Case of Loose Bodies in an Osteo-Arthritic Knee Joint.}

Complaint.-A woman, aged 6I years, complained of weakness in her left knee, with attacks of sudden pain and "giving way" in it. These sharp pains were consistently located to the same place, at the inner side of the patella. They were relieved after a little manipulation, when something was felt to "slip out".

Examination.-Palpation at the painful site revealed a rounded body the size of a hazel nut. It was freely movable, and was probably a loose body or joint mouse. On moving the knees, crepitus was felt in both; it was typical of an osteo-arthritic joint. Further, there was slight limitation of full flexion, but at her age, 6r years, this was within the limits of the normal. In addition, both her feet were flat, with gross hallux valgus and overlying bunions. Her hands also showed nodular joint thickenings and deformity of the finger joints.

X-ray Examination.-In her hand, she held the antero-posterior and lateral radiograms of the affected knee. These showed:-

(a) Two loose bodies (as indicated by the arrows in Fig. I), one to the inner side of the patella, and the other in the supra-patellar pouch.

(b) There was good joint space between the ends of the bones.

Diagnosis.-Two loose bodies in an osteo-arthritic joint, the bodies probably being detached osteophytes. It was not a difficult conclusion.

Comments.-Several candidates failed to make the diagnosis, even after inspection of the radiograms. Others did not look at the radiograms, although the patient held them. It is important to take advantage of all the information offered.

I was further surprised at the casual manner in which the joint was labelled as arthritis, peri-arthritis, rheumatoid arthritis, and osteo-arthritis of the single joint type. The last in particular, as the feet were bare and showed arthritis, and the patient's hands were similarly thickened and deformed from the same affection. Vague terms indicate a hazy clinical appreciation of joint diseases. The Fellowship calls for a high standard.

On enquiring the probable consistence of the loose bodies, cartilage and calcified cartilage were frequently suggested. This was not quite correct. The bodies showed up clearly in the radiograms, and further, the shadow was of the same density as that of the bone. If the loose body were cartilage, no shadow would be shown; if of calcified cartilage, a spiculated shadow darker than that of the bone would be likely. The diagnosis of a loose body in an osteo-arthritic joint, possibly a detached osteophyte, and that it probably consisted of bone, was the correct answer.

Treatment by Operation, 27/II/33.-Immediately before the patient was brought to the theatre, the knee (already surgically prepared), was X-rayed (see Figs. $2 \& 3$ ), and carefully splinted before she was moved from the X-ray table. The great value of this essential pre-operative radiogram will be immediately appreciated on comparing it with that of the knee taken at the original consultation two months previously (Fig. I.).

The knee joint was opened through a longitudinal incision, above and in the line of the lateral border of the patella. Actually, the loose bodies "popped out" on opening the synovial membrane. One of them was quite free, and the other still attached to the synovial membrane. I deduct that the former was the body first seen and felt at the inside of and below the patella. 
There were several other small, sago-like bodies attached to the synovial membrane visible in the joint: these, however, were left undisturbed.

This operation is interesting as it emphasises an important point in the technique of the removal of loose bodies from joints, i.e., an X-ray examination and splinting of the joint immediately before operation; otherwise the bodies may justify their title of "Joint Mice" and be hidden in a corner elsewhere than under the incision.

Conclusion.-Although the diagnosis was straightforward, even without the radiogram, this case was shown to stimulate consideration of such a possible question as, "Discuss the ætiology, pathology, and treatment of loose bodies in joints".

A Brief Description of the Loose Bodies in Joints.-Loose bodies usually occur in the knee and in the elbow joints. They may consist of fibrin, of connective tissue or of bone.

Fibrinous loose bodies are found in tuberculous and osteo-arthritic joints, being described as "melon-seed" bodies in the former. On palpation of such a joint during movement, a characteristic crackling sensation is imparted to the hand. Rarely, they follow a hæmarthrosis in an otherwise normal joint.

\section{Loose bodies of connective tissue and bone.}

(I) In the Osteo-arthritic Joint.-Osteophytic outgrowths are often present in these joints, and owing to their prominence, they may be detached by a trifling injury. The bone at the site of fracture is usually dead. There are seldom more than two or three such loose bodies, and usually only one.

Loose bodies also arise from enlarged pedunculated synovial fringes, and later they may become free in the joint. Such bodies contain fat, cartilage and fibrous tissue.

Somewhat rarely Lipoma Arboresens is seen in a chronic arthritic joint. Here the synovial fringes are very exuberant and fatty but not truly lipomatous. The synovial membrane packs closely into the joint.

(2) In an apparently Normal Joint of a Young Adult Male.-A single body consisting of articular cartilage with an underlying flake of bone becomes detached from the articular surface of the bone, chiefly from the femur, into the knee joint. There is often a history of preceding trauma, and the term "Quiet Necrosis" was fittingly applied to it by Sir James Paget. Sometimes the cartilage proliferates and the body is of considerable size. X-ray examination shows both the detached fragment of bone, and sometimes an irregularity on the contour of the bone-end, which indicates the site of its origin.

(3) In Tuberculous Joints.-Fringes of synovial membranes sometimes separate and give rise to loose bodies of cartilage or fibrous tissue. Further, a triangular sequestrum of bone from the articular surface of the epiphysis occasionally separates into the joint.

(4) Charcot's Joint.-No description of joint loose bodies would be complete without mentioning those not infrequently found in the hypertrophic variety of Charcot's Joint; but seldom do they call for surgical interference. 


\section{A Case of Keith's Diaphyseal Aclasis.}

\section{Alternative Names.}

I. Congenital deforming chondro-dysplasia.

2. Multiple cartilaginous exostoses.

3. Hereditary multiple exostoses.

4. Multiple congenital osteo-chondromata.

Complaint.-The patient, a girl aged ro years, showed exostoses at the upper end of both humeri, at the upper end of both tibiæ, and at the lower end of the right tibia (See Figs. 4, 5, $6 \&$ 7). On the other leg, at the upper tibia and lower fibula were operation scars, apparently for the removal of similar exostoses. In addition, the fibulæ and tibiæ were bowed, and her legs were not the usual length for her size, but were rather short and misshapen.

Family History.-The grandmother, the child's mother, her aunt, her uncle, her first cousin and her brother were all similarly affected.

Diagnosis.-This was a case of Keith's diaphyseal aclasis.

Comments.-The child's mother was present, and in view of the condition being hereditary, I hoped enquiries would be made of her to determine if this congenital factor was present. In no case were such questions asked, except after suggestion that candidates should do so.

A Brief Description of the Condition.-This hereditary disease is about three times as common in men as in women. It manifests itself as multiple growths on the bones, and they are first noticed towards puberty. The deformities or bone pain first call attention to the condition. Almost any bone may be affected, with the rare exception of the face and skull (i.e. bones laid down in membrane). These subjects are often of small stature with deformities of knockknee and Coxa Vara.

The changes observed are:-

(I) Growth retardation.- This affects the form, the length and the thickness of the bones, and it is best seen in the metacarpals on comparing the skiagrams of the hands. The latter bones usually retain their normal length but are thin and delicate. Long bones may show shortening or considerable bowing, probably due to unequal growth of two adjacent bones. Cyst formation is occasionally revealed by $\mathrm{X}$-rays.

(2) Proliferative changes.-These are observed on both sides of the epiphyseal line. The early disappearance of the line itself is a radiological feature. The heads of the bones may show enlargement, defective modelling, and sometimes distinct outgrowths from the epiphysis.

From the shaft of the diaphysis, continuous with the epiphyseal cartilage; arise definite exostoses. They frequently follow an injury. At first, consisting of cartilage, they later ossify, or may even entirely disappear. 
The disease ceases when skeletal development is complete. The essential pathology is that of excessive loss of calcium during the active stages of the disease.

\section{Complications.}

(a) Bursitis in an adventitious bursa overlying an exostosis.

(b) A possible aneurysm arising by erosion of a main artery on the edge of one of the exostoses. I have seen a popliteal aneurysm so caused.

(c) Occasionally a malignant degenerative change occurs in one of the swellings.

Conclusion.-This somewhat rare case was brought for general demonstration and to explore candidates' knowledge of the pathology of the condition. It was an opportunity to correlate book-reading with clinical observation.

I wish to express my appreciative thanks to Dr. Rhynland Parry, Radiologist at the King George Hospital, Ilford, for kindly lending me the radiograms of these two cases.

Case presented by Harold Dodd, M.Ch., F.R.C.S.(Eng.)

\section{Branchial Cyst.}

The patient, a married woman, aged 49 years, presented a localised swelling on the left side of the neck. This swelling had been slowly increasing in size for the past year, was painless and gave rise to no symptoms. There was a previous history (of considerable import but elicited by only a minority of candidates) of a similar swelling 2I years previously and temporarily cured by aspiration.

On examination the swelling was a well-defined oval lump, about the size of a cherry, situated just behind and above the angle of the mandible, and fairly obviously coming from beneath the anterior border of the sternomastoid. (The demonstration of this last sign should surely be within the powers of any F.R.C.S. candidate, and yet several did not know that one should, or how to, put the muscle on the stretch.) On the other hand, the question of fluctuation was almost unanimously decided in the affirmative-a decision which later proved to be perfectly correct, though by no means easy to make, as at subsequent operation the cyst was found to be very thick-walled and very tense. The lack of any definite attachment to either superficial or deep structures was a noteworthy sign.

Most candidates diagnosed, rightly, a branchial cyst. To those who elicited the above important fact in the previous history this was, of course, probably the obvious conclusion though the tensity and height in the neck were a little against the typical example. Tuberculous adenitis was suggested by some candidates, but the mobility after a year's history should have excluded this; malignancy also could be ruled out in view of the consistency of the tumour; lymphadenoma was unlikely in view of the patient's age and the fact of the swelling being solitary over such a lengthy period; a parotid cyst seemed rather far-fetched, as probably did the demonstrator's suggestion of cystic changes in adenomatous aberrant thyroid tissue!

Operation revealed the cyst to be very thick-walled (probably the result of previous aspiration) and tensely filled with a glairy mucoid fluid. There was a suggestion of a stalk attachment to the pharynx, the unobliterated cleft concerned probably being the second. Pathological examination showed a lining of squamous epithelium and very fibroid walls.

Case presented by Arthur E. Porritr, F.R.c.s. 